\title{
Solar coronal magnetic fields derived using seismology techniques applied to omnipresent
} \section{sunspot waves}

\author{
David B. Jess" ${ }^{1,2 \star}$, Veronika E. Reznikova ${ }^{3}$, Robert S. I. Ryans ${ }^{1}$, Damian J. Christian², Peter H. Keys ${ }^{1,4}$, \\ Mihalis Mathioudakis ${ }^{1}$, Duncan H. Mackay ${ }^{5}$, S. Krishna Prasad', Dipankar Banerjee ${ }^{6}$, \\ Samuel D. T. Grant' ${ }^{1}$, Sean Yau' and Conor Diamond ${ }^{1}$
}

Sunspots on the surface of the Sun are the observational signatures of intense manifestations of tightly packed magnetic field lines, with near-vertical field strengths exceeding 6,000 G in extreme cases ${ }^{1}$. It is well accepted that both the plasma density and the magnitude of the magnetic field strength decrease rapidly away from the solar surface, making high-cadence coronal measurements through traditional Zeeman and Hanle effects difficult as the observational signatures are fraught with low-amplitude signals that can become swamped with instrumental noise ${ }^{2,3}$. Magneto-hydrodynamic (MHD) techniques have previously been applied to coronal structures, with single and spatially isolated magnetic field strengths estimated as 9-55 G (refs 4-7). A drawback with previous MHD approaches is that they rely on particular wave modes alongside the detectability of harmonic overtones. Here we show, for the first time, how omnipresent magneto-acoustic waves, originating from within the underlying sunspot and propagating radially outwards, allow the spatial variation of the local coronal magnetic field to be mapped with high precision. We find coronal magnetic field strengths of $32 \pm 5 \mathbf{G}$ above the sunspot, which decrease rapidly to values of approximately $1 \mathrm{G}$ over a lateral distance of $7,000 \mathrm{~km}$, consistent with previous isolated and unresolved estimations. Our results demonstrate a new, powerful technique that harnesses the omnipresent nature of sunspot oscillations to provide magnetic field mapping capabilities close to a magnetic source in the solar corona.

Solar active regions are one of the most magnetically dynamic locations in the Sun's tenuous atmosphere. The intrinsically embedded magnetic fields support a wide variety of coronal compressive MHD wave phenomena, with EUV observations dating back to the late 1990 s revealing a wealth of magnetoacoustic oscillations travelling upwards from the solar surface along magnetic fields to distances far exceeding many tens of thousands of $\mathrm{km}$ (refs 8-11). The weak dissipation mechanisms experienced in the solar corona, in the forms of thermal conduction, compressive viscosity and optically thin radiation ${ }^{12}$, allow features to be probed through seismology techniques in an attempt to uncover atmospheric changes and sub-resolution structuring that would otherwise be difficult, if not impossible to determine ${ }^{13}$. MHD seismology of sunspots is a powerful tool that has risen in popularity in recent years, with insights into atmospheric structuring, expansion and emission gained through the comparison of wave periods, velocities and dissipation rates ${ }^{14,15}$. Importantly, evidence continues to be brought forward that suggests compressive MHD wave phenomena originating in sunspots are not simply a rare occurrence, but instead an omnipresent feature closely tied to all atmospheric layers ${ }^{16}$.

Using previously deployed MHD seismology techniques to infer the strength of the coronal magnetic field is a difficult task, often limited by substantial errors (up to $80 \%$ in some cases ${ }^{4}$ ). Critical problems are its inability to deduce the magnetic field strength as a function of spatial position, and by the relative rareness of oscillatory phenomena in the solar corona demonstrating measurable harmonic overtones ${ }^{7}$. Other methods, including deep exposures of coronal magnetic Stokes profiles ${ }^{17}$ and the examination of brightness-temperature spectra from gyro-resonance emission ${ }^{18}$, also bring about their own distinct impediments, including crosstalk between Stokes signals, poor temporal resolutions often exceeding $1 \mathrm{~h}$ and ambiguous cyclotron frequency reference points ${ }^{19}$. Therefore, although diverse, yet encouraging attempts have been made to constrain the magnitude of the coronal magnetic field in previous years, each process is hindered by its inability to accurately and simultaneously deduce spatially resolved, unambiguous and high-cadence field strengths. In this Letter we document and apply a novel MHD-based technique that combines high-resolution observations from modern space-based observatories, vector magnetic field extrapolations and differential emission measure techniques to accurately measure the spatial dependence of the coronal magnetic field strength with potential temporal resolutions orders-of-magnitude better than current imaging approaches.

Observations at high spatial $(435 \mathrm{~km}$ per pixel) and temporal (12s) resolution with the Atmospheric Imaging Assembly ${ }^{20}$ (AIA) on-board the Solar Dynamics Observatory (SDO) spacecraft have allowed us to resolve fine-scale and rapidly dynamic coronal features associated with a powerful magnetic sunspot. The data were obtained through six independent EUV filters on 10 December 2011 , with the resulting fields of view cropped to $50 \times 50 \mathrm{Mm}^{2}$ to closely match simultaneous ground-based observations obtained with the Rapid Oscillations in the Solar Atmosphere ${ }^{21}$ (ROSA)

\footnotetext{
${ }^{1}$ Astrophysics Research Centre, School of Mathematics and Physics, Queen's University Belfast, Belfast BT7 1NN, UK. ${ }^{2}$ Department of Physics and Astronomy, California State University Northridge, Northridge, California 91330, USA. ${ }^{3}$ Center for Mathematical Plasma Astrophysics, Department of Mathematics, KU Leuven, Celestijnenlaan 200 B bus 2400, B-3001 Heverlee, Belgium. ${ }^{4}$ Solar Physics and Space Plasma Research Centre (SP2 RC), University of Sheffield, Hicks Building, Hounsfield Road, Sheffield S3 7RH, UK. ${ }^{5}$ School of Mathematics and Statistics, University of St Andrews, St Andrews KY16 9SS, UK. ${ }^{6}$ Indian Institute of Astrophysics, II Block, Koramangala, Bangalore 560 034, India. ${ }^{*}$ e-mail: d.jess@qub.ac.uk
} 
a

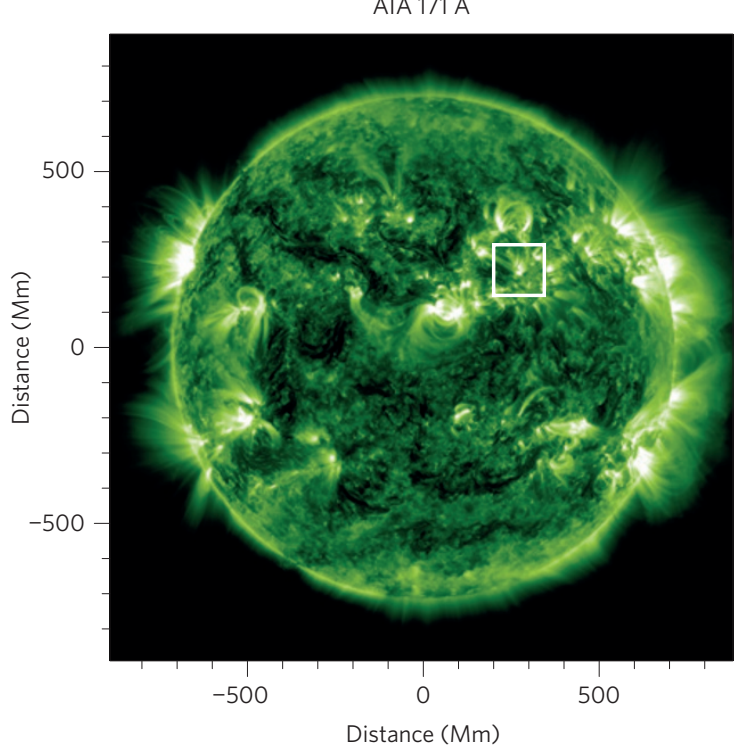

b

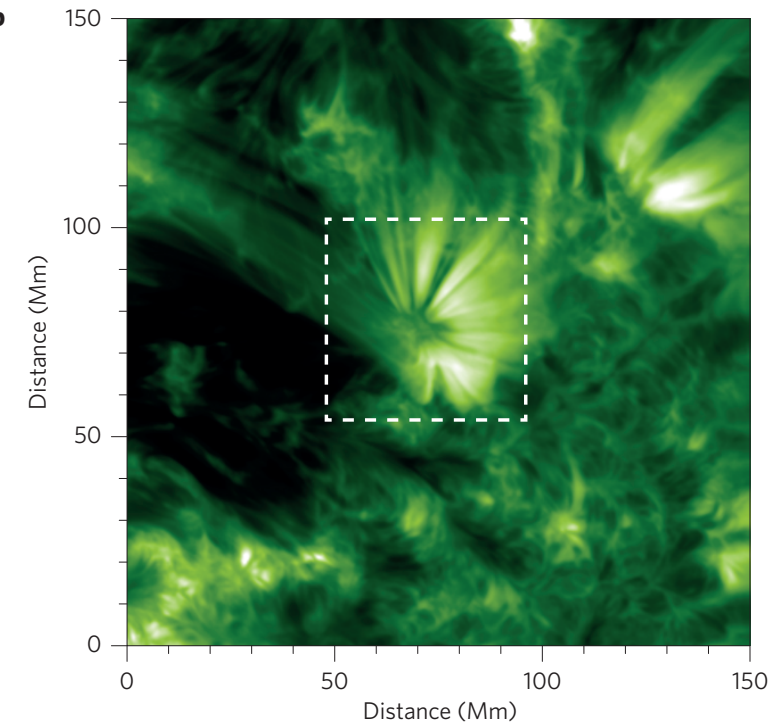

c

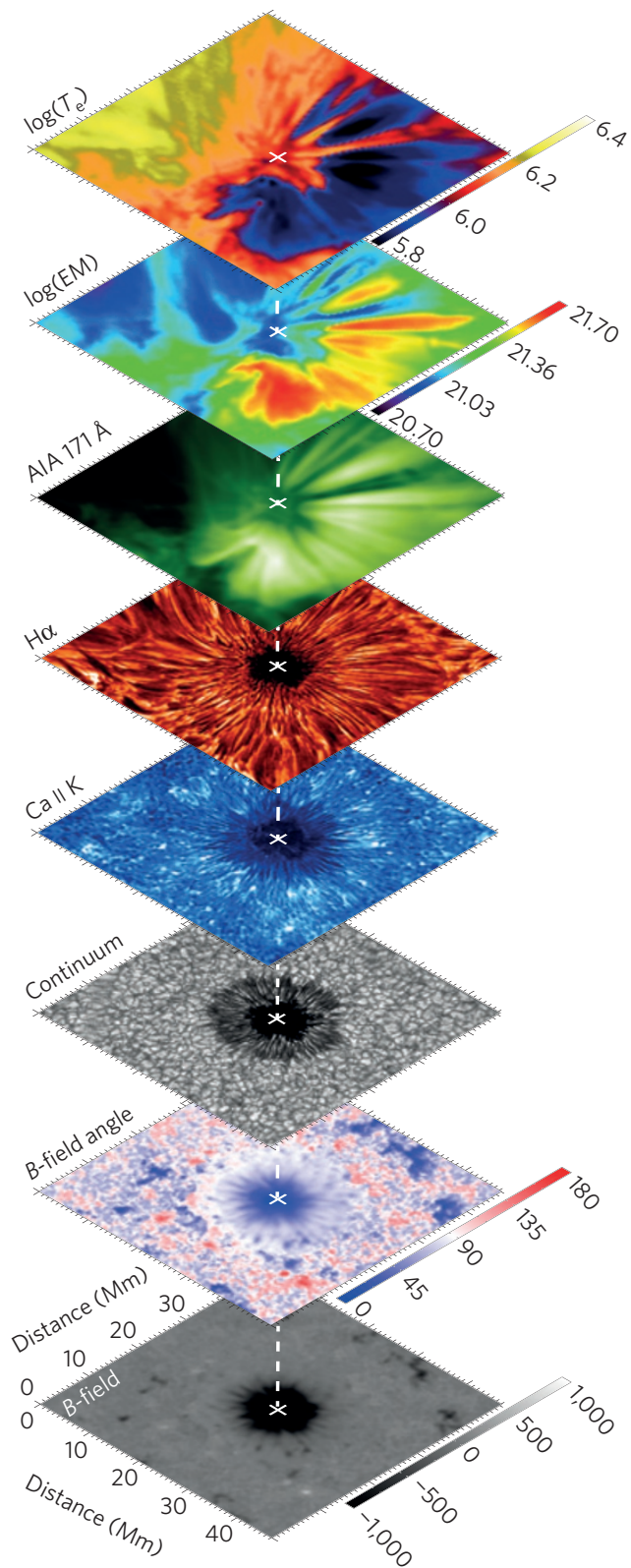

Figure 1 | The building blocks of the magnetized solar atmosphere observed on 10 December 2011. a, A contextual EUV $171 \AA$ image of the entire Sun on 10 December 2011 at 16:10 UT, where a solid white box highlights a $150 \times 150 \mathrm{Mm}^{2}$ subfield surrounding NOAA 11366. b, A zoom-in to the subfield outlined in a, with a further $50 \times 50 \mathrm{Mm}^{2}$ subfield highlighted using dashed white lines to show the pointing and size of the ground-based field of view. c, Co-spatial images, stacked from the solar surface (bottom) through to the outer corona (top). From bottom to top the images consist of snapshots of the photospheric magnetic field strength normal to the solar surface $\left(B_{z}\right.$; artificially saturated at $\pm 1,000 \mathrm{G}$ to aid clarity), the inclination angles of the photospheric magnetic field with respect to the normal to the solar surface $\left(0^{\circ}\right.$ and $180^{\circ}$ represent magnetic field vectors aligned downwards and upwards, respectively, to the solar surface), ROSA 4,170 $\AA$ continuum intensity, ROSA Ca II K lower chromospheric intensity, HARDcam H $\alpha$ upper chromospheric intensity, coronal AIA $171 \AA$ intensity, emission measure (on a log-scale in units of $\mathrm{cm}^{-5} \mathrm{~K}^{-1}$ ) and kinetic temperature (shown on a log-scale artificially saturated between $\log \left(T_{\mathrm{e}}\right)=5.8$ and $\log \left(T_{\mathrm{e}}\right)=6.4$ to assist visualization). White crosses mark the location of the umbral barycentre and are interconnected as a function of atmospheric height using a dashed white line.

and Hydrogen-Alpha Rapid Dynamics camera ${ }^{14}$ (HARDcam) instruments on the Dunn Solar Telescope (see Supplementary Methods for further information on the processing of the observational data). The images (Fig. 1) show a number of elongated structures extending away from the underlying sunspot, which are particularly apparent in AIA $171 \AA$ data known to correspond to lower coronal plasma with temperatures $\sim 700,000 \mathrm{~K}$. Differential emission measure (DEM) techniques ${ }^{22}$ are employed on the six EUV AIA channels, producing emission measure (EM) and temperature $\left(T_{\mathrm{e}}\right)$ estimates for the sunspot and its locality. The resulting $T_{\mathrm{e}}$ map reveals that the elongated structures, seen to extend away from the underlying sunspot, are comprised of relatively cool coronal material $(<1,000,000 \mathrm{~K})$, hence explaining why they demonstrate such visibility in the AIA $171 \AA$ channel. Examination of time-lapse movies (Supplementary Movie 1) reveals a multitude of wavefronts seen to propagate radially outwards from the sunspot, particularly along the relatively cool, elongated coronal fan structures.

Fourier-filtered images (Fig. 2 and Supplementary Movie 2) reveal a wealth of oscillatory signatures covering a wide range of frequencies, with longer periodicities preferentially occurring 
a

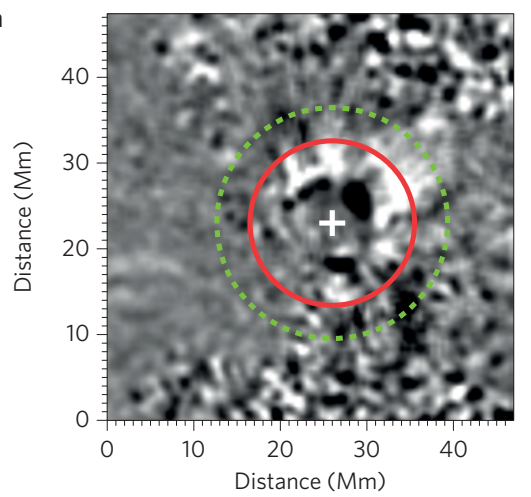

b

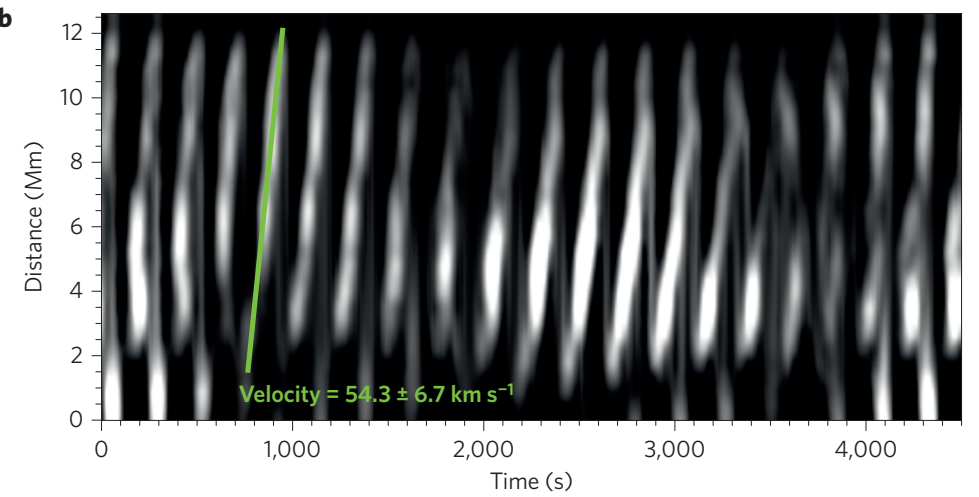

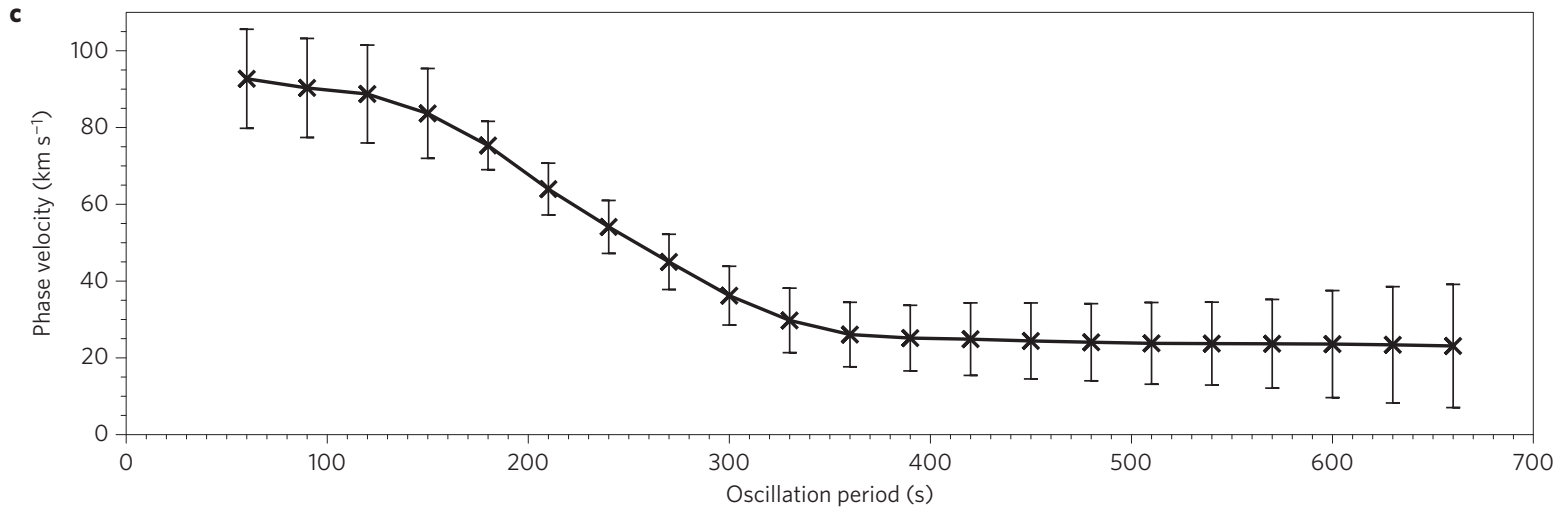

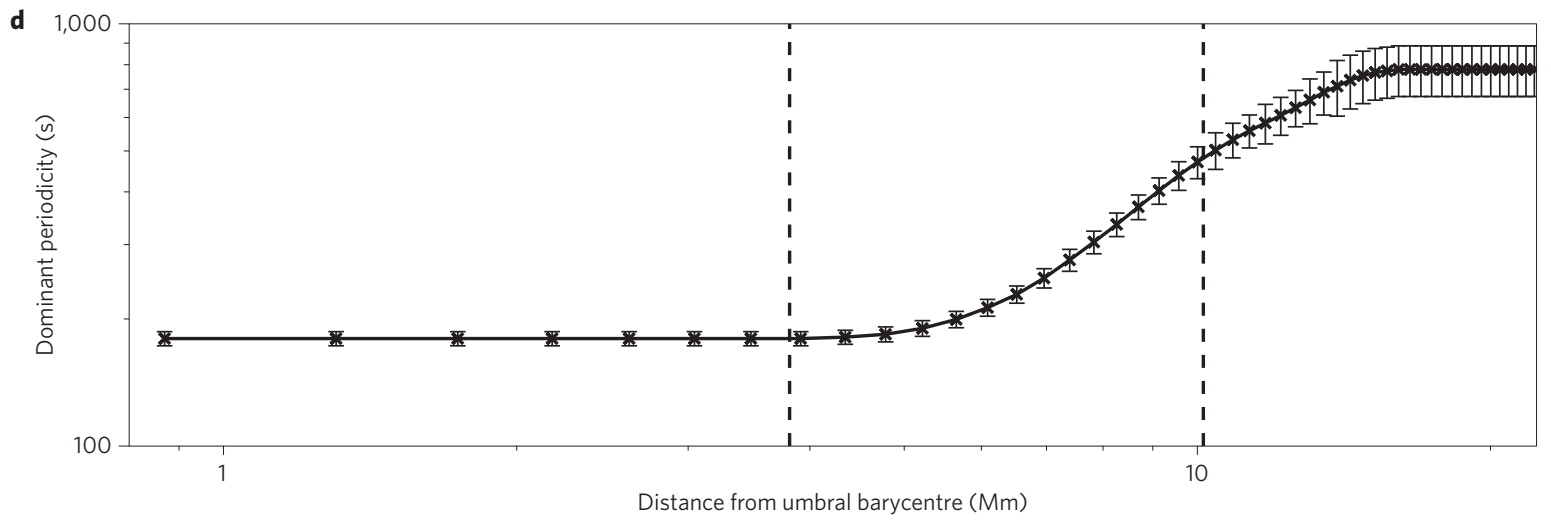

Figure 2 | Measured velocities and periodicities in the vicinity of a sunspot. a, A snapshot of a $171 \AA$ intensity image having first been passed through a 4-min temporal filter. The umbral barycentre is marked with a white cross, whereas the instantaneous peaks and troughs of propagating waves are revealed as white and black intensities, respectively, immediately surrounding the sunspot (see also Supplementary Movie 2 ). The solid red line highlights an expanding annulus, whereas the dotted green line outlines the entire spatial extent used in the creation of the time-distance diagram (b), whereby the area delimited by the dotted green curve is azimuthally averaged into a one-dimensional line, directed radially away from the umbral barycentre. $\mathbf{b}$, The resulting time-distance diagram, consisting of 30 spatial (approximately $13 \mathrm{Mm}$ ) by 375 temporal ( 75 min) pixels ${ }^{2}$. A solid green line highlights a line of best fit used to calculate the period-dependent phase speeds. c, The period-dependent phase speeds (in $\mathrm{km} \mathrm{s}^{-1}$ ), following the Fourier filtering of the time series, plotted as a function of oscillatory period. The error bars show the standard deviations associated with the minimization of the sum of the squares

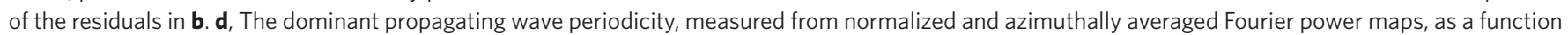
of radial distance from the underlying umbral barycentre. The error bars relate to the Fourier frequency resolution of the SDO/AIA instrument operating with a cadence of $12 \mathrm{~s}$. The vertical dashed lines represent the umbral and penumbral boundaries established from photospheric SDO/HMI continuum images at approximately 3.8 Mm and 10.1 Mm, respectively, from the centre of the sunspot umbra.

at increasing distances from the sunspot umbra, and propagating with reduced phase speeds. Importantly, the waves are apparently continuous in time, suggesting that such oscillatory phenomena are driven from a regular, powerful and coherent source. Through centre-of-gravity techniques the centre of the sunspot (or umbral barycentre) is defined, as indicated by the white crosses in Fig. 1c. A series of expanding annuli, centred on the umbral barycentre, are used to map the azimuthally averaged phase velocities as a function of period through the creation of time-distance diagrams (Fig. 2).
Phase speeds are calculated from the gradients of the diagonal bands in the time-distance diagrams and found to span $92 \mathrm{~km} \mathrm{~s}^{-1}$ (60 s periodicity) through to $23 \mathrm{~km} \mathrm{~s}^{-1}$ ( $660 \mathrm{~s}$ periodicity), which are characteristic of coronal slow magneto-acoustic tube speeds ${ }^{9-12}$, $c_{\mathrm{t}}$. Owing to the continuous nature of the propagating waves, Fourier methods are applied to the unfiltered data to create power maps allowing the signatures of the wave phenomena to be easily extracted as a function of period. Employing the same expanding annuli technique, the period-dependent power spectra are studied 
a

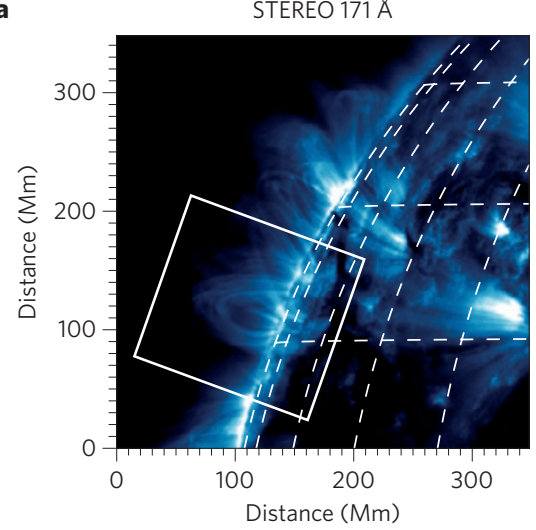

b

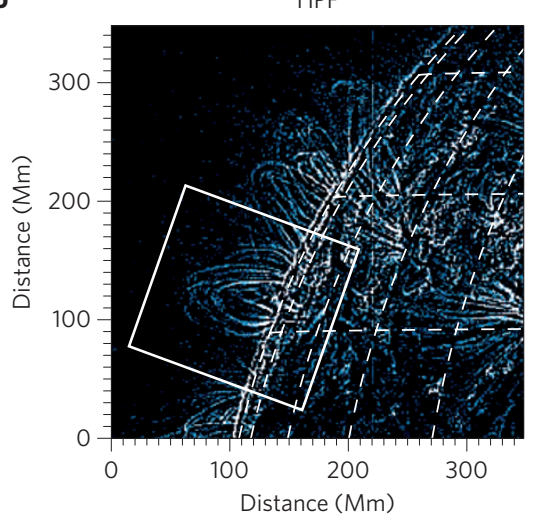

C

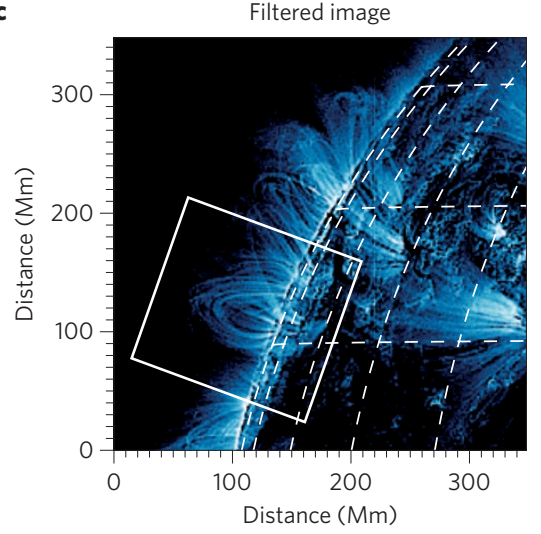

d

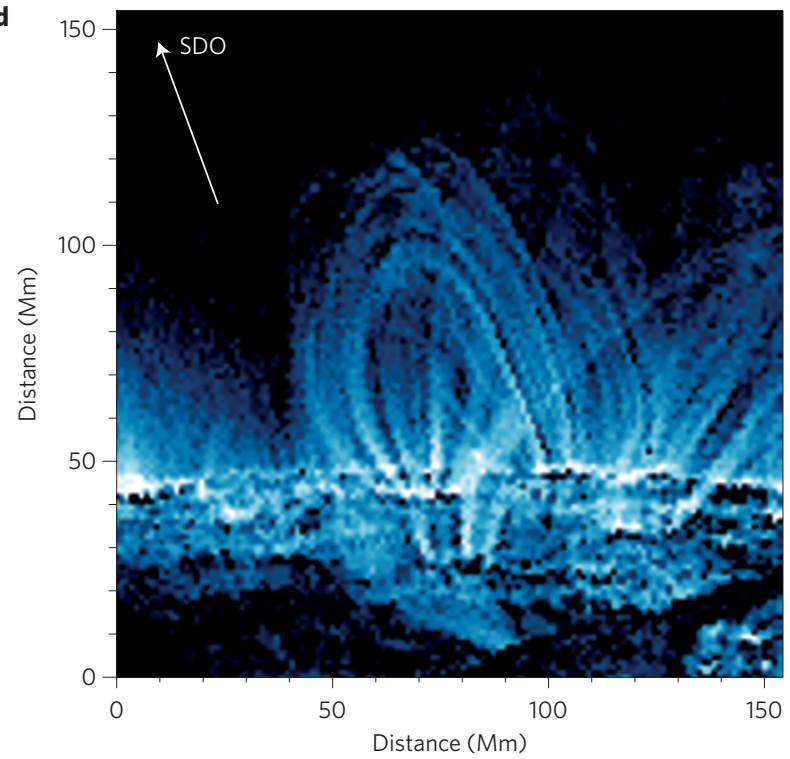

e

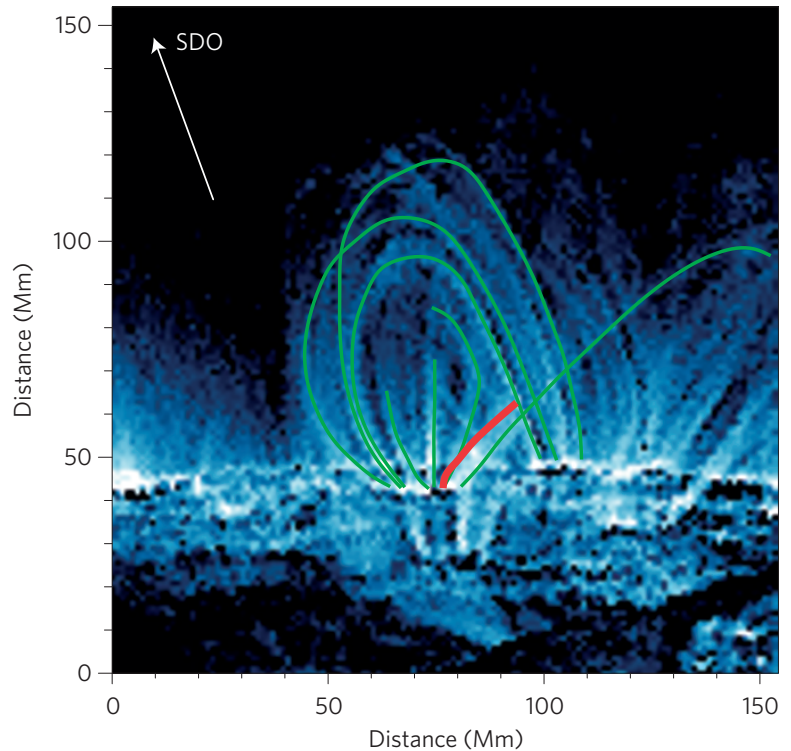

Figure 3 | A side view of the coronal fan from filtered STEREO-A images. a, A 171 $\AA$ image acquired by the STEREO-A spacecraft at 16:14 UT on 10 December 2011, where white dashed lines indicate solar heliographic coordinates with each vertical and horizontal line separated by $10^{\circ}$. The solid white box highlights the region extracted for measurements in $\mathbf{d}, \mathbf{e}$. $\mathbf{b}$, The STEREO-A image having been run through a high-pass filter to reveal small-scale

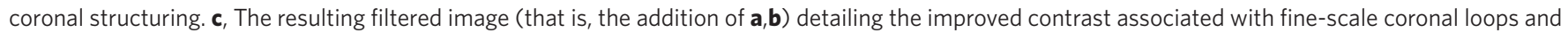
fans. $\mathbf{d}$, A zoom-in to the region highlighted by the white boxes in a-c and rotated so the solar limb is horizontal. The white arrow indicates the direction

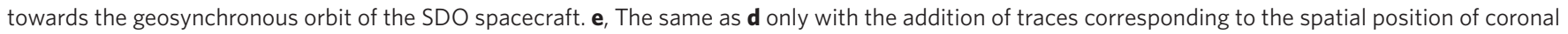
loops in the immediate vicinity of NOAA 11366 (green lines) and the coronal fan at present under investigation (red line).

as a function of the radial distance from the umbral barycentre (Supplementary Fig. 1). A gradual change in the dominant period as a function of distance is found, with periodicities of approximately 3 min within the underlying sunspot umbra, increasing rapidly within the penumbral locations, and eventually plateauing at periodicities of approximately 13 min further along the coronal fan structures (Fig. 2d). This suggests that the waves detected within the immediate periphery of the underlying sunspot are the lower coronal counterpart of propagating compressive magneto-acoustic oscillations driven upwards by the underlying sunspot.

Complementary $171 \AA$ data from the EUVI instrument onboard the leading Solar TErrestrial RElations Observatory ${ }^{23}$ (STEREO-A) provides a side-on view of the coronal fan, allowing the spatial variation of the structure with atmospheric height to be measured directly (Fig. 3). Magnetic field extrapolations ${ }^{24}$ of vector magnetograms from SDO's Helioseismic and Magnetic Imager ${ }^{25}$ (HMI) are also used to calculate magnetic field vectors over a wide range of atmospheric heights, allowing field inclination angles to be compared with direct STEREO-A imaging as a function of distance from the umbral barycentre (Fig. 4). We find that increasing the distance from the centre of the sunspot results in more inclined magnetic fields. Importantly, this means that the measured phase speeds of the propagating waves will have been underestimated to a certain degree, particularly for locations close to the umbral barycentre, where the magnetic field inclination is minimal. To compensate for this, we first use the velocity-period and perioddistance relationships to convert the measured tube speeds into a function of distance from the centre of the underlying sunspot (Fig. 4). Then, the inclination angles at this spatial position are used to calculate the true wave velocity irrespective of inclination-angle effects (Fig. 4d). Tube speeds of approximately $185 \mathrm{~km} \mathrm{~s}^{-1}$ are found towards the centre of the umbra, decreasing radially within several Mm to values of approximately $25 \mathrm{~km} \mathrm{~s}^{-1}$.

Because the sound speed, $c_{s}$, is dependent on the local plasma temperature, we use the $T_{\mathrm{e}}$ maps to derive the spatial distribution of the sound speed through the relation

$$
c_{\mathrm{s}}=\sqrt{\frac{\gamma R T_{\mathrm{e}}}{\mu}}
$$


a

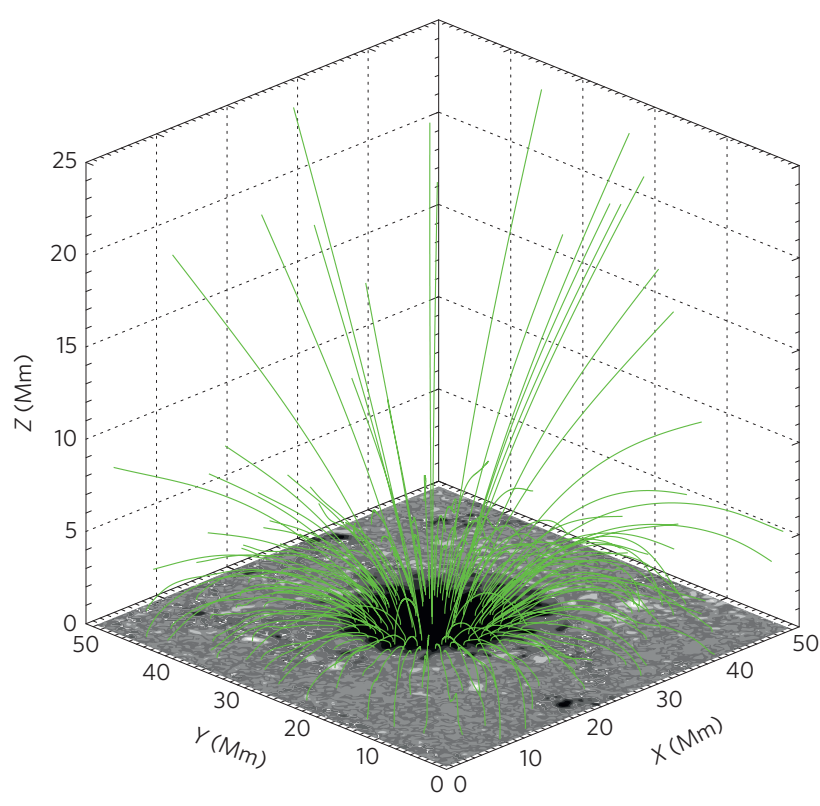

b

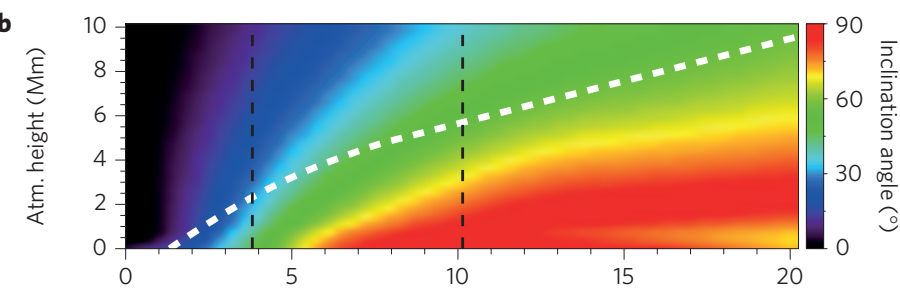

c

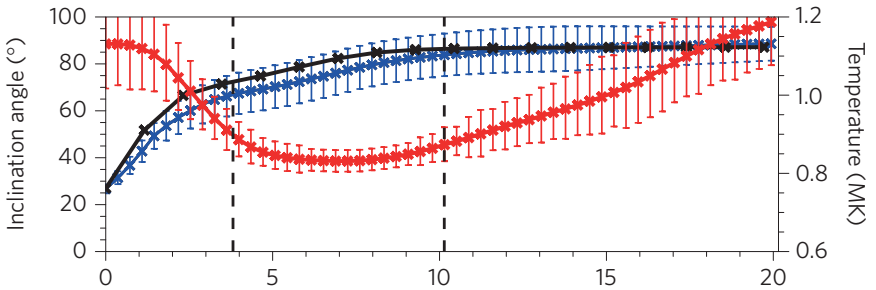

d

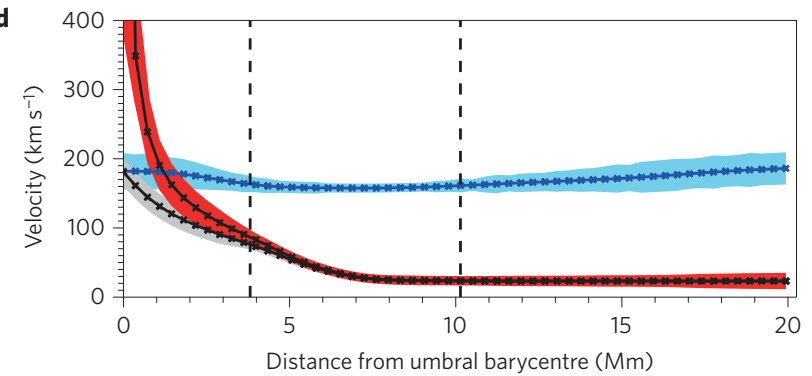

Figure 4 | Magnetic, temperature and velocity structuring of the sunspot plasma. a, The extrapolated magnetic fields (green lines) overlaid on the photospheric $B_{z}$ map, and viewed from an angle of $45^{\circ}$ to the solar surface. $\mathbf{b}$, The azimuthally averaged inclination angles of the extrapolated magnetic field vectors with respect to the normal to the solar surface, shown as a function of both the distance from the umbral barycentre and the atmospheric height. Here, $0^{\circ}$ and $90^{\circ}$ are angles parallel and perpendicular, respectively, to the normal to the solar surface. The dashed white line overlays the coronal fan position as observed by STEREO-A $171 \AA$ images. c, The inclination angles of the coronal fan measured from STEREO-A images (black line) alongside the extrapolated magnetic field inclination angles (blue line) corresponding to the dashed white line in $\mathbf{b}$. Both sets of inclination angles have been transposed into the viewing perspective of the SDO spacecraft by taking into account the location of SDO with respect to the normal to the solar surface. Error bars show the standard deviations associated with the azimuthal averaging of the inclination angles contained within each corresponding annuli. A considerable agreement between the measured and extrapolated field inclination angles demonstrates the accuracy of potential force-free magnetic extrapolations in this instance. The azimuthally averaged plasma temperature (in MK) derived from DEM techniques is shown using a red line, where the error bars represent the temperature widths associated with DEM fitting techniques. $\mathbf{d}$, The measured tube speed ( $c_{\mathrm{t}}$; black line with grey error contours) following compensation from inclination-angle effects to provide the true tube speed irrespective of angle ambiguities, the temperature-dependent sound speed ( $c_{s}$; blue line with light blue error contours) calculated from the local plasma temperature, and the subsequently derived Alfvén speed ( $v_{\mathrm{A}}$; black line with red error contours) shown as a function of distance from the underlying umbral barycentre. Errors in the sound speeds and tube speeds are propagated from the temperature width and a combination of inclination angle (c) plus Fourier frequency resolution (Fig. 2d) plus residual phase velocity measurement (Fig. 2c) errors, respectively. The errors associated with the Alfvén speeds result from the combination of sound speed and tube speed error estimates. The vertical dashed lines represent the umbral and penumbral boundaries established from photospheric SDO/HMI continuum images at approximately $3.8 \mathrm{Mm}$ and $10.1 \mathrm{Mm}$, respectively, from the centre of the sunspot umbra.

where $\gamma=5 / 3$ is the adiabatic index, $\mu=1.3 \mathrm{~g} \mathrm{~mol}^{-1}$ is the mean molecular weight assuming $\mathrm{H}: \mathrm{He}=10: 1$ for the solar corona $^{26}$, and $R$ is the gas constant. This provides sound speeds greater than the compensated tube speeds, and of the order of $160-210 \mathrm{~km} \mathrm{~s}^{-1}$ (Fig. 4d). With the distributions of $c_{\mathrm{s}}$ and $c_{\mathrm{t}}$ known, we estimate the spatial distribution of the Alfvén speed, $v_{\mathrm{A}}$, through the relationship ${ }^{27}$

$$
v_{\mathrm{A}}=\frac{c_{\mathrm{s}} c_{\mathrm{t}}}{\sqrt{c_{\mathrm{s}}^{2}-c_{\mathrm{t}}^{2}}}
$$

The derived Alfvén speeds, shown in Fig. 4d, are highest $\left(1,500 \mathrm{~km} \mathrm{~s}^{-1}\right)$ towards the centre of the sunspot umbra, dropping to their lowest values $\left(25 \mathrm{~km} \mathrm{~s}^{-1}\right)$ at the outermost extremity of the active region. The large values found towards the sunspot core are consistent with previous coronal fast-mode wave observations related to magnetically confined structures ${ }^{28}$.

The Alfvén speed depends on two key parameters: the magnitude of the magnetic field strength, $|B|$, and the local plasma density, $\rho$. We calculate ${ }^{22}$ spatially resolved plasma densities from the EM and $T_{\mathrm{e}}$ maps, resulting in a range of densities of the order of
(2.1-5.6) $\times 10^{-12} \mathrm{~kg} \mathrm{~m}^{-3}$. With the Alfvén speeds and local plasma densities known, we derive the magnitude of the azimuthally averaged magnetic field at each radial location through the relationship ${ }^{27}$

$$
|B|=v_{\mathrm{A}} \sqrt{\mu_{0} \rho}
$$

where $\mu_{0}$ is the magnetic permeability. The resulting values for $|B|$ range from $32 \pm 5 \mathrm{G}$ at the centre of the underlying sunspot, rapidly decreasing to $1 \mathrm{G}$ over a lateral distance of approximately 7,000 km (Fig. 5a). Both our maximal and minimal values are consistent with previous, yet isolated and/or errorprone independent estimations of magnetically dominated coronal loops $^{4-7}$ and quiet Sun locations ${ }^{29}$. However, importantly, we show for the first time a novel way of harnessing the omnipresent nature of propagating slow-mode waves in the corona to more accurately constrain the magnetic field topology as a function of spatial location, with the potential to provide coronal $B$-field maps with cadences as high as $1 \mathrm{~min}$. This would provide more than an orderof-magnitude improvement in temporal resolution compared with deep exposures of coronal Stokes profiles ${ }^{17}$. A key science goal of 

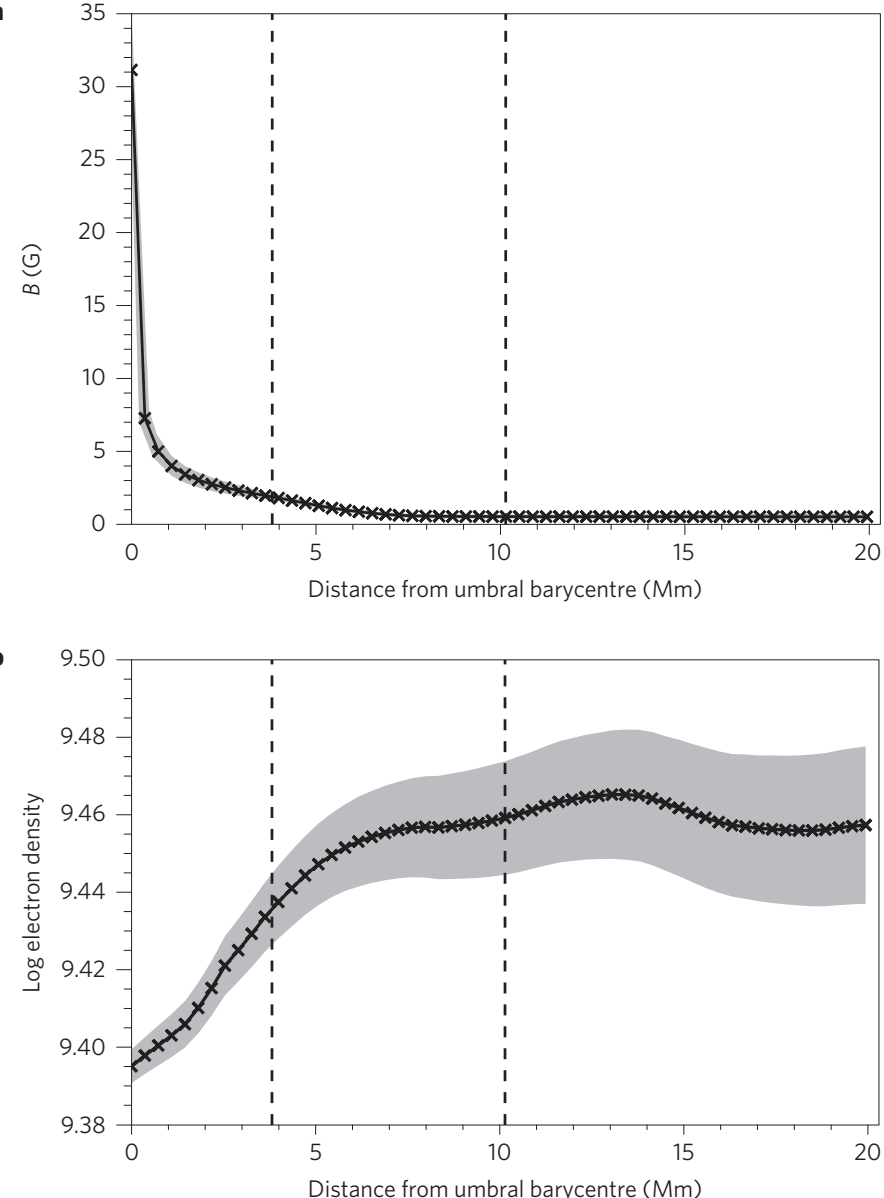

c

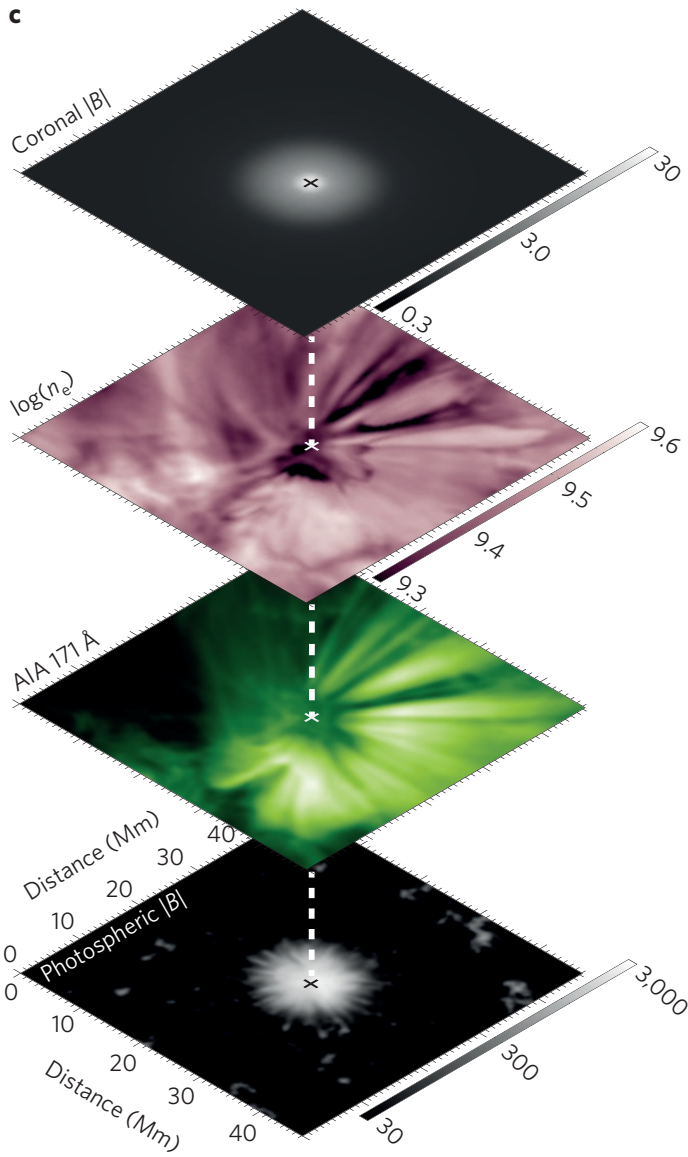

Figure $\mathbf{5}$ | The magnitude of the magnetic field in the solar corona. $\mathbf{a}$, The magnitude of the magnetic field strength, $|B|$, plotted as a function of radial distance from the umbral barycentre, where the grey error shading represents the propagation of errors associated with the Alfvén speeds (Fig. 4d) and the plasma densities (b). b. The azimuthally averaged electron densities, $n_{\mathrm{e}}$, plotted on a log-scale (units of $\mathrm{cm}^{-3}$ ) using the same distance scale as $\mathbf{a}$, where the vertical dashed lines represent the umbral and penumbral boundaries established from photospheric SDO/HMI continuum images at approximately 3.8 $\mathrm{Mm}$ and $10.1 \mathrm{Mm}$, respectively, from the centre of the sunspot umbra. Greyscale error shading highlights the goodness of fit that is defined by a least-squares optimization of the plasma emission measures using DEM approaches. c, Co-spatial images providing (from bottom to top): a two-dimensional representation of the magnitude of the photospheric magnetic field strength (displayed on a log-scale and saturated between 30 and $3,000 \mathrm{G}$ to aid clarity); the EUV AIA $171 \AA$ intensity; the electron densities (saturated between $\log \left(n_{\mathrm{e}}\right)=9.3$ and $\log \left(n_{\mathrm{e}}\right)=9.6$ to assist visualization); and the reconstructed magnitude of the coronal magnetic field strength (shown circularly symmetric on a log-scale and saturated between 0.3 and $30 \mathrm{G}$ to aid clarity). White and black crosses indicate the position of the umbral barycentre, which are connected between atmospheric heights using a dashed white line.

the upcoming $4 \mathrm{~m}$ Daniel K. Inouye Solar Telescope (DKIST) is to directly image the full coronal Stokes profiles using near-infrared spectral lines. The novel techniques presented here will therefore serve as an important long-term tool that can be used to compare directly with the DKIST observations following first light in 2019. Furthermore, because magnetic reconnection phenomena are often observed in the vicinity of active regions, a high-cadence approach to monitoring the spatial variations of the coronal magnetic field would be critical when attempting to understand the precursor events leading to solar flares.

Received 15 September 2014; accepted 6 October 2015; published online 16 November 2015

\section{References}

1. Livingston, W., Harvey, J. W., Malanushenko, O. V. \& Webster, L. Sunspots with the strongest magnetic fields. Sol. Phys. 239, 41-68 (2006).

2. Merenda, L., Trujillo Bueno, J., Landi Degl'Innocenti, E. \& Collados, M. Determination of the magnetic field vector via the Hanle and Zeeman effects in the He I $\lambda 10830$ multiplet: Evidence for nearly vertical magnetic fields in a polar crown prominence. Astrophys. J. 642, 554-561 (2006).
3. Kramar, M., Inhester, B. \& Solanki, S. K. Vector tomography for the coronal magnetic field. I. Longitudinal Zeeman effect measurements. Astron. Astrophys. 456, 665-673 (2006).

4. Nakariakov, V. M. \& Ofman, L. Determination of the coronal magnetic field by coronal loop oscillations. Astron. Astrophys. 372, L53-L56 (2001).

5. Verwichte, E., Nakariakov, V. M., Ofman, L. \& Deluca, E. E. Characteristics of transverse oscillations in a coronal loop arcade. Sol. Phys. 223, 77-94 (2004).

6. Van Doorsselaere, T., Nakariakov, V. M. \& Verwichte, E. Coronal loop seismology using multiple transverse loop oscillation harmonics. Astron. Astrophys. 473, 959-966 (2007).

7. Van Doorsselaere, T., Nakariakov, V. M., Young, P. R. \& Verwichte, E. Coronal magnetic field measurement using loop oscillations observed by Hinode/EIS. Astron. Astrophys. 487, L17-L20 (2008).

8. Deforest, C. E. \& Gurman, J. B. Observation of quasi-periodic compressive waves in solar polar plumes. Astrophys. J. 501, L217-L220 (1998).

9. Ofman, L., Nakariakov, V. M. \& Deforest, C. E. Slow magnetosonic waves in coronal plumes. Astrophys. J. 514, 441-447 (1999).

10. De Moortel, I. \& Hood, A. W. The damping of slow MHD waves in solar coronal magnetic fields. Astron. Astrophys. 408, 755-765 (2003).

11. Krishna Prasad, S., Banerjee, D. \& Van Doorsselaere, T. Frequency-dependent damping in propagating slow magneto-acoustic waves. Astrophys. J. 789, 118 (2014). 
12. De Moortel, I. \& Bradshaw, S. J. Forward modelling of coronal intensity perturbations. Sol. Phys. 252, 101-119 (2008).

13. Wang, T. J. et al. Hot coronal loop oscillations observed with SUMER: Examples and statistics. Astron. Astrophys. 406, 1105-1121 (2003).

14. Jess, D. B. et al. The source of 3 minute magnetoacoustic oscillations in coronal fans. Astrophys. J. 757, 160 (2012).

15. Yuan, D., Sych, R., Reznikova, V. E. \& Nakariakov, V. M. Multi-height observations of magnetoacoustic cut-off frequency in a sunspot atmosphere. Astron. Astrophys. 561, A19 (2014).

16. Jess, D. B., Reznikova, V. E., Van Doorsselaere, T., Keys, P. H. \& Mackay, D. H The influence of the magnetic field on running penumbral waves in the solar chromosphere. Astrophys. J. 779, 168 (2013).

17. Lin, H., Penn, M. J. \& Tomczyk, S. A new precise measurement of the corona magnetic field strength. Astrophys. J. 541, L83-L86 (2000).

18. Gary, D. E. \& Hurford, G. J. Coronal temperature, density, and magnetic field maps of a solar active region using the Owens Valley Solar Array. Astrophys. J. 420, 903-912 (1994).

19. White, S. M. \& Kundu, M. R. Radio observations of gyroresonance emission from coronal magnetic fields. Sol. Phys. 174, 31-52 (1997).

20. Lemen, J. R. et al. The atmospheric imaging assembly (AIA) on the solar dynamics observatory (SDO). Sol. Phys. 275, 17-40 (2012).

21. Jess, D. B. et al. ROSA: A high-cadence, synchronized multi-camera solar imaging system. Sol. Phys. 261, 363-373 (2010).

22. Aschwanden, M. J., Boerner, P., Schrijver, C. J. \& Malanushenko, A. Automated temperature and emission measure analysis of coronal loops and active regions observed with the atmospheric imaging assembly on the Solar Dynamics Observatory (SDO/AIA). Sol. Phys. 283, 5-30 (2013).

23. Kaiser, M. L. et al. The STEREO mission: An introduction. Space Sci. Rev. 136, 5-16 (2008).

24. Guo, Y. et al. Modeling magnetic field structure of a solar active region corona using nonlinear force-free fields in spherical geometry. Astrophys. J. 760, 47 (2012).

25. Schou, J. et al. Design and ground calibration of the helioseismic and magnetic imager (HMI) instrument on the solar dynamics observatory (SDO). Sol. Phys. 275, 229-259 (2012)
26. Aschwanden, M. J. Observations and models of coronal loops: from Yohkoh to TRACE SOLMAG 2002-Proceedings of the Magnetic Coupling of the Solar Atmosphere Euroconference and IAU Colloquium 188 ESA Publications Division, Vol. 505, 191-198 (2002).

27. Roberts, B. Waves and oscillations in the corona-(Invited Review). Sol. Phys. 193, 139-152 (2000)

28. Yuan, D. et al. Distinct propagating fast wave trains associated with flaring energy releases. Astron. Astrophys. 554, A144 (2013).

29. Long, D. M., Williams, D. R., Régnier, S. \& Harra, L. K. Measuring the magnetic-field strength of the quiet solar corona using "EIT Waves". Sol. Phys. 288, 567-583 (2013)

\section{Acknowledgements}

D.B.J. wishes to thank the UK Science and Technology Facilities Council (STFC) for the award of an Ernest Rutherford Fellowship in addition to a dedicated Research Grant. D.B.J. also wishes to thank Invest NI for their Research \& Development Grants. This work was supported by a UKIERI trilateral research grant of The British Council.

\section{Author contributions}

D.B.J., V.E.R., S.K.P., S.Y. and C.D. performed analysis of observations. D.B.J., M.M., S.K.P. and D.B. interpreted the observations. D.B.J., P.H.K., S.Y., R.S.I.R., D.J.C., S.D.T.G. and C.D. performed all image processing. D.B.J., D.H.M., D.J.C. and M.M. designed the observing run. All authors discussed the results and commented on the manuscript.

\section{Additional information}

Supplementary information is available in the online version of the paper. Reprints and permissions information is available online at www.nature.com/reprints. Correspondence and requests for materials should be addressed to D.B.J.

\section{Competing financial interests}

The authors declare no competing financial interests. 